\title{
Ambiente de moradia e controle primário em idosos
}

\author{
Hilma Tereza Tôrres Khoury \\ Universidade Federal do Pará, Belém-PA, Brasil \\ Isolda de Araújo Günther \\ Universidade de Brasília, Brasília-DF, Brasil
}

Resumo: Este trabalho investigou a relação entre ambiente de moradia e duas modalidades de controle percebido - controle primário (CP) e controle secundário (CS), referentes a esforços empreendidos para adaptar o ambiente às próprias necessidades e realizar metas $(\mathrm{CP})$; adaptar-se ao ambiente (CS). Participaram 315 idosos (105 homens; 210 mulheres) residentes em BrasíliaDF, entre 60 e 92 anos, selecionados de forma não-probabilística, por cotas de gênero. Os dados foram coletados no domicílio por meio de entrevista incluindo questões sócio-demográficas e uma escala de controle. Os resultados apontam: (1) Relação inversa entre CP e densidade social do ambiente de moradia - DESAM $(r=-0,119 ; \mathrm{p} \leq 0,05)$; (2) Percepção de controle significativamente maior nos que dispunham de quarto exclusivo $(\mathrm{t}=2,21 ; \mathrm{df}=313 ; \mathrm{p} \leq 0,05)$. Conclui-se que baixa DESAM facilita o CP nessa fase da vida em que muitas atividades são realizadas no domicílio, convertendo a liberdade de ação e a privacidade em necessidades importantes.

Palavras-chave: Psicologia ambiental. Psicologia do desenvolvimento. Moradia. Idosos.

\section{Home environment and primary control in elderly people}

\begin{abstract}
The relation between home environment and two modalities of perceived control: primary control (PC) and secondary control (SC), was investigated. PC and SC refer to efforts exerted to: a) adapt the environment to the residents' needs and attainment of goals; $b$ ) adapt to the environment. The non-probabilistic quota sample was composed of 315 residents in BrasíliaDF, Brazil, (105 male; 210 female), between 60 and 92 years old. Data were collected through interviews at the respondents' households. Results indicated: 1) Inverse correlation between PC and social density of the home environment - DESAM $(r=-0.119 ; \mathrm{p} \leq 0.05) ; 2)$ Perceived control was significantly higher among elderly who had their own bedroom $(t=2.21 ; \mathrm{df}=313 ; \mathrm{p}$ $\leq 0.05)$ compared to those who did not have an exclusive bedroom. The results suggest that low DESAM favors PC in this stage of life, when many activities are performed at home, and freedom of action and privacy are very important needs.
\end{abstract}

Keywords: Environmental psychology. Developmental psychology. Housing. Aged.

\section{Ambiente de vivienda y control primario en ancianos}

Resumen: Se investigó la relación entre ambiente de vivienda y dos modalidades de control percibido: control primario (CP) y control secundario (CS), referentes a esfuerzos emprendidos para adaptar el ambiente a las propias necesidades y realizar metas (CP); adaptarse al ambiente (CS). Participaron 315 ancianos (105 hombres; 120 mujeres) residentes en Brasilia - DF, entre 60 y 92 años, seleccionados de manera no probabilística, por cuotas de género. Los datos fueron colectados en la dirección por medio de entrevista incluyendo cuestiones socio-demográficas y una escala de control. Los resultados apuntan: 1) Relación inversa entre CP y densidad social del ambiente de vivienda - DESAM ( $\mathrm{r}=$ $0,119 ; \mathrm{p} \leq 0,05) ; 2$ ) percepción de control significativamente mayor en los que disponían de habitación exclusiva $(\mathrm{t}=2,21 ; \mathrm{df}=313 ; \mathrm{p} \leq 0,05)$. Se concluyó que baja DESAM facilita el CP en esa etapa de la vida en que muchas actividades son realizadas en la dirección, convirtiendo la libertad de acción y la privacidad en necesidades importantes.

Palabras clave: Psicología ambiental. Psicología del desarrolo. Morada. Adultos mayores. 
A Psicologia Ambiental e a Psicologia do Desenvolvimento têm demonstrado a relação interativa entre os processos de desenvolvimento e os contextos em que se vive. Por sua vez, a Gerontologia Social destaca o papel do ambiente físico quando do declínio das habilidades funcionais, ao chamar atenção para a importância do ambiente de moradia no processo de envelhecimento. O planejamento e a adaptação da casa, espaço de vida, tanto favorecem o exercício das capacidades funcionais nas atividades da vida diária (Lawton, 1983; Perracini, 2006; Pynoos, Nishita \& Perelman, 2003), quanto proporcionam segurança e bem-estar a idosos frágeis, dependentes ou com demência (Lerner, 2001; Perracini, 2006).

É nesse sentido que Lawton (1983), pioneiro no estudo da relação entre envelhecimento e ambiente, enfatizou que as preocupações primárias dos idosos estão relacionadas à manutenção do controle, da independência e da privacidade. Corroborando esse princípio, a literatura psicológica tem explicitado os efeitos produzidos no com-portamento e nos afetos pela percepção de controle pessoal ou por sua falta (Seligman \& Csikszentmihalyi, 2000).

Lawton (1983) também adverte acerca dos efeitos negativos de ambientes com elevada densidade social, isto é, grande número de pessoas num determinado espaço físico, sobre o comportamento humano, demonstrando sua atuação na perda do controle social. Esses dados sugeriram ainda que, acreditar-se detentor, ou não, de controle influencia o impacto que a elevada densidade social do ambiente tem sobre as pessoas (Bell, Greene, Fisher \& Baum, 2001; Sinhá \& Nayyar, 2000).

Revendo teorias acerca dos efeitos negativos da alta densidade social no comportamento humano, Bell e cols. (2001) afirmaram que tais efeitos "causam uma redução no controle" (p. 315). Por exemplo, na noção de restrição do comportamento, a alta densidade atua "eliminando opções de comportamento e reduzindo a liberdade para se comportar como se quer" (p. 316). Nos modelos de regulação da privacidade, segundo os quais o controle do grau de intimidade nas interações pessoa-pessoa se dá pelo ajustamento da distância entre uma pessoa e outra, um ambiente de densidade muito elevada pode privar o sujeito de qualquer escolha. "Dessa maneira, podemos perder o controle sobre a regulação da intimidade" (Bell e cols., 2001, p. 316). Esses dados não implicam que os idosos devam viver apartados, que as gerações devam viver isoladas umas das outras. De fato, nos anos tardios a co-residência com os filhos adultos constitui um dos mais freqüentes arranjos habitacionais.

Os efeitos da alta densidade social foram minimizados por Oliver (2002), quando afirmou que, diferentemente dos animais em cativeiro, os humanos têm maneiras de escapar. Sugeriu como mecanismos de escape "desde tomar um trem ou um carro e viajar (...) se mudar (...) até construir uma extensão da casa" (p. 73). Convém lembrar que essas possibilidades envolvem mobilidade e finanças, dois recursos nem sempre disponíveis à população em geral.

Os efeitos diferenciais da alta densidade na saúde física, mental e no comportamento social foram retomados por Oliver (2002) ao apresentar o modelo densidade-intensidade formulado por Freedman (conforme citado por Oliver, 2002). De acordo com esse modelo, em qualquer situação, se a pessoa tem um sentimento positivo ou negativo em relação ao ambiente, a alta densidade o intensificará. Ao examinar esse modelo, no que se refere às variações da densidade social de moradias, Duckitt (1983) confirmou essa associação apenas no que se refere ao afeto negativo. Sentimentos positivos não foram aumentados ou reduzidos em função da maior densidade da moradia.

Sinha e Nayyar (2000) relataram achados similares, ao mostrarem que os idosos residentes em ambientes de alta densidade apresentaram dificuldade em manter níveis desejados de privacidade e controle, além de avaliarem seus ambientes de moradia mais negativamente que os residentes em ambientes de baixa densidade. Esses autores visavam estudar o impacto da percepção de controle pessoal no sentido de amortecer os efeitos estressantes da elevada densidade no ambiente de moradia. No entanto, a interação entre controle pessoal e densidade social não foi estatisticamente significativa. Assim, concluíram que a alta densidade do ambiente de moradia é tão aversiva que a percepção de seus efeitos estressantes não pode ser melhorada pela percepção de elevado controle pessoal. 
O presente artigo considera a convergência entre teorias da Psicologia Ambiental, da Psicologia do Desenvolvimento e da Gerontologia Social, agregando o conceito de densidade social. Analisase a influência de variáveis do ambiente de moradia sobre a percepção de controle em idosos.

O trabalho é um recorte de uma pesquisa mais ampla, cujo objetivo foi investigar o controle primário e o controle secundário em idosos, buscando-se relações com variáveis sócio-demográficas e com alguns indicadores de envelhecimento bem-sucedido (Khoury, 2005). Entre as variáveis demográficas estudadas incluiu-se o ambiente de moradia.

Controle primário $(\mathrm{CP})$ e controle secundário (CS) são formas de controle pessoal percebido. O primeiro se refere a todos os esforços que o indivíduo realiza para adaptar o ambiente - físico ou social às suas necessidades e realizar suas metas. O controle secundário refere-se aos esforços que o indivíduo empreende para se adaptar ao ambiente e "dançar conforme a música" (Khoury \& Günther, 2006). Esses esforços podem ser em nível de ação/ comportamento ou de cognição/pensamento. Nesse estudo, o controle primário foi avaliado em dois aspectos: esforço de realização com recursos próprios e esforço de realização com recursos externos (ajuda de outras pessoas ou de tecnologias). O controle secundário foi considerado como esforços dirigidos para a adaptação a situações em que metas não foram alcançadas ou onde ocorreram perdas/falhas.

$\mathrm{O}$ ambiente de moradia foi estudado por meio das seguintes variáveis: densidade social do ambiente de moradia, designada por DESAM e definida conforme Gifford (2007); quarto exclusivo (só para si ou só para o casal); presença/ausência de elevador na residência e piso/andar em que reside o idoso. A densidade social do ambiente de moradia foi calculada pela seguinte fórmula:

DESAM $=\left[\left(\mathrm{N}^{\circ}\right.\right.$. de residentes na moradia $/ \mathrm{N}^{\circ}$. de quartos $)+\left(\mathrm{N}^{\mathrm{o}}\right.$. de residentes na moradia/ $\mathrm{N}^{\circ}$. de banheiros)] /2. A DESAM foi conceituada a partir da formulação do IBGE (Instituto Brasileiro de Geografia e Estatística) que calcula densidade por meio da relação entre número de moradores e número de quartos. Neste estudo acrescentou-se outro fator, número de banheiros.
Procurou-se responder às seguintes perguntas: (1) A DESAM influencia a percepção do controle primário em idosos? (2) As crenças e os comportamentos de controle são afetados por outras variáveis do ambiente de moradia? Com base na revisão da literatura, duas hipóteses foram levantadas neste estudo: (1) o controle primário seria maior entre os idosos que vivem em ambientes com menor densidade social; (2) o controle primário seria maior entre aqueles que possuem maior privacidade (quarto exclusivo), bem como entre os que residem em ambientes que facilitam a saída de casa, por exemplo, morar em casa ou nos andares mais baixos; ter elevador no prédio em que reside.

\section{Método}

\section{Amostra}

Participaram 315 idosos (105 homens e 210 mulheres), residentes em Brasília-DF, com idades entre 60 e 92 anos (Média = 71,3 anos; DP =6,9), selecionados de forma não-probabilística, porém por cotas de gênero aproximadas às existentes na população (41,6\% de homens e 58,4\% de mulheres). Em média, os participantes possuíam 11,1 anos de escolaridade, acumulavam uma renda familiar mensal de $\mathrm{R} \$ 5.043,64$ e renda per capita de $\mathrm{R} \$ 2.077,93$.

Entre as características do ambiente de moradia da amostra cabe destacar:

a) Número de residentes na casa/apartamento: Média =3,11; $\mathrm{DP}=1,62$ (amplitude de variação $=1$ a 9);

b) DESAM: Média $=1,12 ; \mathrm{DP}=0,69$ (amplitude de variação $=0,18$ a 5,33);

c) Quarto Exclusivo: $\mathrm{Sim}=93,7 \%$; Não = 6,3\%; 43,5\%;

d) Elevador na residência: $\mathrm{Sim}=56,5 \%$; Não $=$

e) Piso/Andar em que reside: $3^{\circ}$ andar e acima $=42,9 \% ; 1^{\circ}$ andar $=29,5 \% ; 2^{\circ}$ andar $=22,9 \%$; Casa $/$ Térreo $=4,8 \%$.

f) Índice de controle primário, referente à soma dos pontos da escala (0-4 pontos; 25 itens): Média $=$ 33,46; DP = 6,76 (amplitude de variação = 15 a 44).

\section{Instrumentos}

Os instrumentos consistiram em uma escala de controle (ECOPSE), especialmente construída e 
validada (Cronbach's alpha $=0,80)$ para a pesquisa que deu origem a esse artigo, com o objetivo de medir controle primário e controle secundário, além de um questionário que solicitava informações sóciodemográficas. A ECOPSE (Khoury, 2005) compõese de 25 itens distribuídos em três fatores: (1) controle primário com recursos próprios - auto-motivação e empenho concreto para alcançar metas ( 11 itens. Ex. Quando realmente quero alguma coisa, faço todo o possível para conseguir; Quando tenho um objetivo, procuro pensar que sou capaz de alcançar.); (2) controle primário com recursos externos (5 itens. Exemplo: Quando não consigo fazer algo sozinho, eu peço ajuda a alguém; Quando eu não consigo compreender alguma coisa, pergunto a quem sabe); (3) controle secundário ( 9 itens. Exemplo: Se o que eu quero fica difícil de alcançar, procuro uma coisa mais modesta; Quando planejo uma coisa e não consigo realizar, eu penso: "Vai ver sonhei alto demais!"). Os par-ticipantes avaliaram os itens por meio de uma escala de cinco pontos, tipo Likert ( 0 = nada a ver comigo; $4=$ tudo a ver comigo $)$.

\section{Procedimentos}

Os potenciais participantes foram procurados em quadras residenciais da cidade de Brasília-DF. Todos os dados foram coletados pela primeira autora, em sessão única com duração média de uma hora, por meio de entrevista individual, realizada no domicílio do idoso. As entrevistas foram conduzidas após consentimento precedido por exposição acerca dos objetivos e importância da pesquisa (Termo de Consentimento Livre e Esclarecido). Inicialmente solicitavam-se informações sócio-demográficas tais como idade, escolaridade, dados referentes ao trabalho e à moradia, religião, renda individual e familiar. Prosseguia-se com questões referentes à rede de apoio social, às condições/limitações relacionadas à saúde, mobilidade, administração da casa e da conta bancária. Em seguida a pesquisadora lia cada item da escala de controle, todos formados por frases afirmativas, e registrava as respostas em uma ficha.

\section{Análise dos dados}

Para verificar a relação entre os controles primário e secundário e a DESAM, assim como entre essas variáveis de controle e o piso (andar) da residência em que habitava o participante, foi calculado o coeficiente produto momento de Pearson. Uma regressão linear foi conduzida a fim de determinar o efeito da DESAM sobre o controle primário com recursos próprios. Para comparar aqueles que possuíam/não possuíam quarto exclusivo, bem como os que dispunham/não dispunham de elevador na residência com respeito ao índice de controle primário, foi utilizado o teste $t$ de Student para amostras independentes. A fim de verificar a existência de associação entre as variáveis categóricas ter/não ter quarto exclusivo e o índice de controle primário alto, médio ou baixo foi realizado o quiquadrado e calculado o $\mathrm{V}$ de Cramer, recurso estatístico para verificar associação entre variáveis categóricas. Adotou-se para todas as provas o nível de significância convencional de 0,05 .

\section{Resultados}

Os resultados apontaram relação inversa entre o controle primário com recursos próprios e a densidade social do ambiente de moradia-DESAM ( $\mathrm{r}=-0,119 ; p \leq 0,05)$. Correlação fraca, porém estatisticamente significativa, indicando que o controle primário é menor naqueles que residem em ambientes com elevada densidade social. A análise de regressão indicou que, para cada unidade de aumento na DESAM, o índice de controle primário com recursos próprios diminuía 1,16 unidades $[\mathrm{DP}=0,12$; variância explicada $=1,1 \% ; F=4,50(1,313) ; p \leq 0,05]$. O controle primário com recursos externos e o controle secundário não mostraram associação significativa com quaisquer das variáveis ambientais.

As variáveis densidade social do ambiente de moradia (DESAM) e controle primário com recursos próprios foram consideradas também em categorias: (a) DESAM Baixa (até 2,0) e DESAM Alta (> 2,0); (b) Baixo Controle (índice $\leq 29$ ), Controle Moderado (índice de 30 a 35) e Alto Controle (índice $\geq 36$ ). O critério para definir a baixa e a alta DESAM tomou como base dados fornecidos pela PNAD (Pesquisa Nacional por Amostra de Domicílios) de 2001 acerca da densidade de moradores por dormitório em área urbana, bem como a média da DESAM na amostra investigada $(1,12)$. De acordo com os dados da PNAD/2001, no Brasil a densidade é igual a 1 para 
19,2\% dos domicílios particulares permanentes; entre mais de 1 e 2 para 56,6\%; entre mais de 2 e 3 para $17 \%$ e entre mais de 3 para $7,2 \%$ dos domicílios. No Distrito Federal, a densidade é igual a 1 para 23,7\% dos domicílios particulares permanentes; entre mais de 1 e 2 para 50,4\%; entre mais de 2 e 3 para $16,1 \%$ e mais de 3 para 9,8\% dos domicílios. O critério para determinar controle baixo, moderado ou alto baseouse na média da amostra e na amplitude de variação. $\mathrm{Na}$ análise de freqüência destas categorias, por meio de tabela cruzada, verificou-se que $43,2 \%$ daqueles que residiam em ambientes com elevada densidade social demonstraram baixa percepção de controle. Por outro lado, $41,4 \%$ dos que residiam em ambientes com baixa densidade social apresentaram elevada percepção de controle (Figura 1).

No que diz respeito às variáveis dicotômicas "quarto exclusivo" e "elevador na residência" foi realizado o teste t para averiguar diferenças quanto aos índices de controle. Os participantes que possuíam quarto exclusivo revelaram maior percepção de controle primário com recursos próprios (Média = 33,$7 ; \mathrm{DP}=6,8$ ) do que aqueles que não tinham um quarto só para si ou para o casal (Média $=30,3$; DP $=5,8)$. A diferença entre as médias foi de 3,4. O teste $\mathrm{t}$ para amostras independentes indicou ser improvável que este resultado tenha ocorrido por erro amostral $(\mathrm{t}[313]=2,21, p \leq 0,05)$. Com relação à variável elevador na residência não houve diferença significativa no índice de controle primário. Na análise de freqüência das variáveis controle primário com recursos próprios (baixo/moderado/alto) e quarto exclusivo ( $\mathrm{sim} /$ não), por meio de tabela cruzada, a Figura 2 aponta que $42,7 \%$ daqueles que possuíam quarto exclusivo demonstraram percepção de controle primário elevada, contra $15 \%$ no grupo dos que não tinham quarto exclusivo. Em contrapartida, $55 \%$ destes se encontravam entre os que evidenciaram baixa percepção de controle primário Tal relação entre o controle primário com recursos próprios e a exclusividade do quarto mostrou-se significativa $\left(\mathrm{X}^{2}=8,74 ; p \leq 0,05 ; \mathrm{gl}=2\right)$. Computouse o V de Cramer, cujo resultado foi um coeficiente de 0,17 e variância explicada $=2,9 \%$. Este resultado indica que privacidade, na forma de um quarto exclusivo, afeta a percepção de controle primário com recursos próprios.

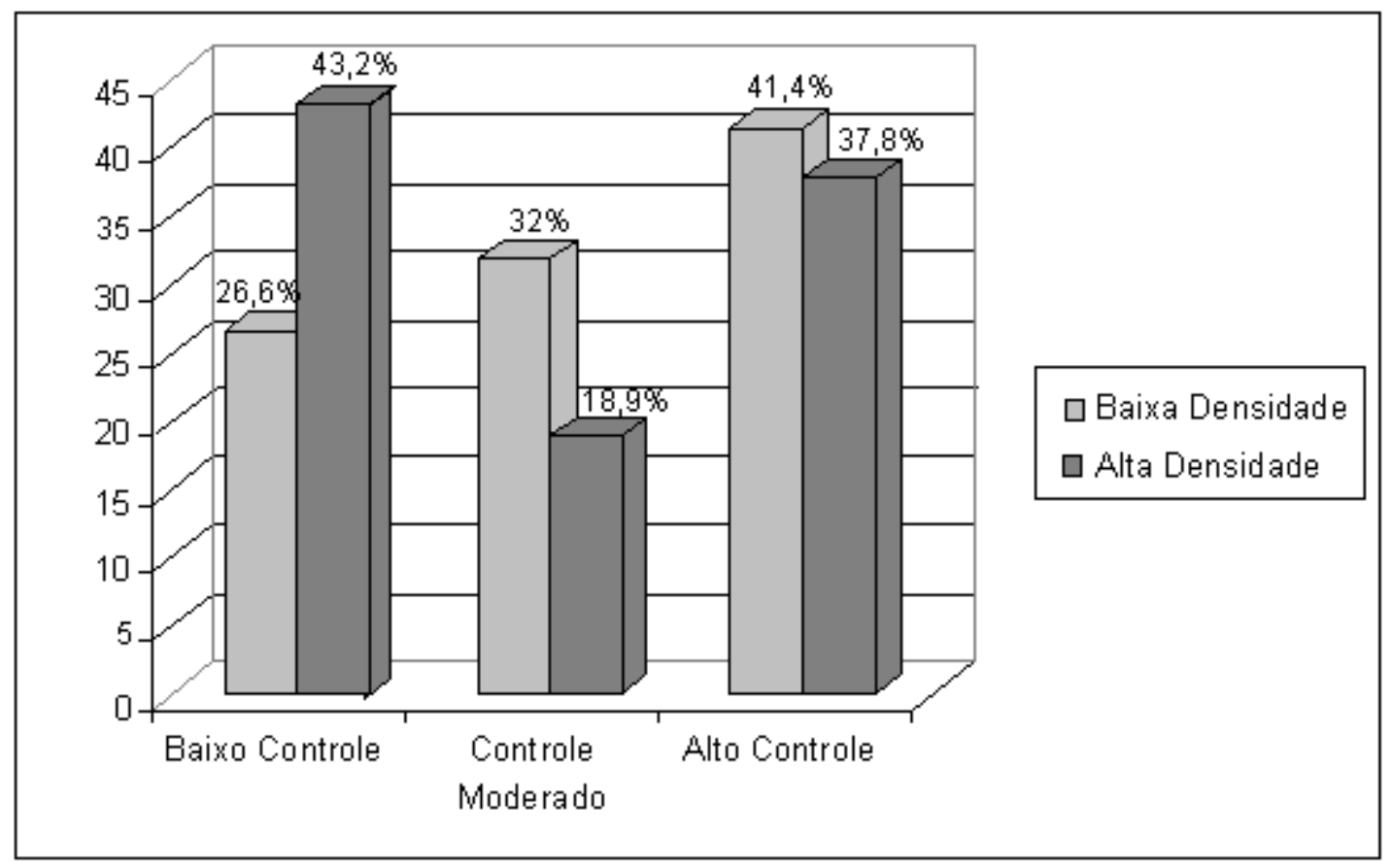

Figura 1. Relação entre densidade social (baixa/alta) do ambiente de moradia e percepção de controle primário (baixo/moderado/alto). 


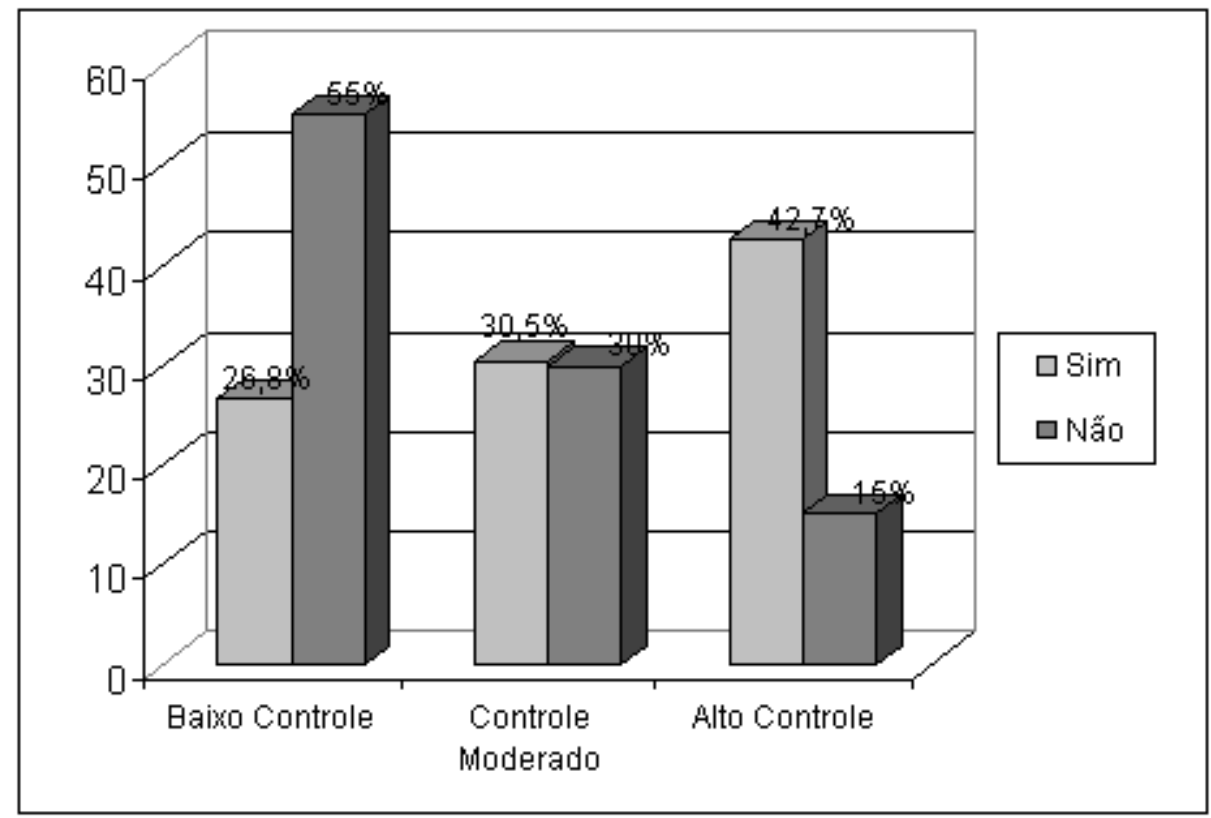

Figura 2. Relação entre exclusividade do quarto (sim/não) e percepção de controle primário (baixo/moderado/alto).

\section{Discussão}

Os resultados desta pesquisa apontaram relação entre densidade do ambiente de moradia e percepção de controle. O controle primário com recursos próprios foi menor entre os idosos que viviam em ambientes com elevada densidade social, confirmando a primeira hipótese e respondendo positivamente à primeira questão investigada. Neste sentido, os resultados desta pesquisa são congruentes com o estudo de Sinha e Nayyar (2000), que indicou que os participantes que moravam em ambientes de alta densidade relataram dificuldade em manter níveis desejados de controle pessoal. Além disso, estão em consonância com a literatura que aponta relação entre alta densidade e perda de controle (Bell e cols., 2001).

A segunda hipótese foi parcialmente confirmada. $\mathrm{O}$ fato de possuir um quarto exclusivo - só para si ou só para o casal - está associado à maior controle primário com recursos próprios. No entanto, contrariamente ao esperado, aqueles que moravam em casa térrea ou nos andares mais baixos revelaram menor controle primário do que aqueles que moravam nos andares mais altos. É provável que aqueles que têm maior controle primário no sentido de mobilidade, se mantenham nos andares mais altos por confiarem mais em sua própria capacidade de adequação ao ambiente. E, por outro lado, é possível que aqueles que têm mais dificuldade para exercer o controle primário procurem os andares mais baixos justamente por isso. Vale notar que o andar em que mora o participante não mostrou correlação significativa com o controle primário com recursos externos, nem com o controle secundário. Destaca-se também que, o fato de ter ou não ter elevador na residência não evidenciou diferença significativa em quaisquer dos tipos de estratégia de controle investigados.

Conclui-se que a baixa densidade social do ambiente de moradia facilita o controle primário nesta fase da vida, em que muitas das atividades são realizadas dentro da casa, convertendo a liberdade de ação, a autonomia e a privacidade em necessidades importantes (Lawton, 1983; Pynoos e cols., 2003). Um ambiente de moradia com baixa densidade social, bem como o fato de se ter um quarto só para si ou só para o casal podem facilitar o controle primário, na medida em que proporcionam maior liberdade e privacidade ao idoso, favorecem uma série de atividades dentro da casa como, por exemplo, 
assistir ao canal de televisão desejado, decidir sobre a própria alimentação, receber amigos, enfim fazer as próprias escolhas.

Como apoio ao fato de que a baixa densidade social favorece o controle primário ou de que a alta densidade social inibe o controle primário, ressaltase que, os residentes em ambientes de elevada densidade eram os que tinham menos amigos, os menos ativos no sentido de participarem de atividades fora da casa, os mais dependentes. Comparados com aqueles que viviam em ambientes de baixa densidade, os idosos que residiam em ambientes de elevada densidade social saíam menos de casa; não participavam de atividades fora da casa visando integração social (exemplo: encontrar amigos), cuidado de si próprio (exemplo: fazer hidroginástica) ou prestar ajuda aos outros (exemplo: realizar trabalho voluntário); não movimentavam conta bancária; dependiam mais dos outros para sair de casa e tinham menos amigos. Além disso, freqüentavam menos a sua igreja ou templo. É como se a elevada densidade social os imobilizasse.

Poder-se-ia argumentar que o fato de morar em ambiente familiar de alta densidade conduziria à maior dependência e inatividade entre idosos; afinal, presume-se que havendo mais pessoas em casa, há mais pessoas com as quais poderiam contar. Contudo, um dado que chama atenção neste estudo é que não se obteve apoio para tal afirmação, desde que a densidade social não revelou correlação significativa com a variável rede de apoio social. Estes resultados fortalecem a literatura acerca dos efeitos da densidade social sobre o comportamento social. Bell e cols. (2001) demonstraram que a elevada densidade "causa retraimento e menos comportamento de ajuda em uma variedade de situações" (p. 309). Em uma revisão de literatura, acerca da relação entre a exposição crônica a ambientes de alta densidade e sintomas de desamparo aprendido, Bell e cols. (2001) concluíram que "à medida que as pessoas em situações de elevada densidade abandonaram suas crenças de que podiam controlar seus ambientes, seus comportamentos crescentemente se tornaram semelhantes aos associados ao desamparo aprendido" (p. 318). Assim, a alta densidade teria maior ou menor impacto sobre as pessoas dependendo de suas crenças de controle: "os efeitos da alta densidade são pelo menos parcialmente determinados pelas percepções de controle das pessoas" (p.319). Entretanto, vale lembrar que, em uma investigação sobre o impacto da percepção de controle pessoal no sentido de amortecer os efeitos estressantes da elevada densidade no ambiente de moradia, Sinha e Nayyar (2000) não encontraram associação significativa entre controle pessoal e densidade ambiental. Assim, concluíram que a alta densidade do ambiente de moradia é tão aversiva que a percepção de seus efeitos estressantes não pôde ser melhorada pela percepção de elevado controle pessoal.

No presente estudo, o controle primário com recursos próprios foi significativamente menor nos que residiam em ambientes com elevada densidade social. O controle secundário não mostrou correlação significativa com esta variável. Portanto, sugestivas evidências parecem indicar que os idosos que residem em ambientes com elevada densidade social não contam sequer com o controle secundário como recurso compensatório para lhes ajudar a manter suas crenças de controle, driblando os efeitos negativos da alta densidade sócio-ambiental. Arrisca-se a dizer que, neste contexto, um ambiente de elevada densidade social associado à falta de controle de onde e como se vive pode ser potencialmente desfavorável a um envelhecimento saudável. Face à realidade socioeconômica e cultural dos participantes deste estudo, refletida no estilo de vida, nas expectativas e exigências, bem como na possibilidade de atendê-las, uma réplica desta investigação está sendo conduzida em uma capital da região norte do Brasil, objetivando comparar os resultados das duas localidades.

\section{Referências}

Bell, P. A., Greene, T. C., Fisher, J. D., \& Baum, A. (2001). Environmental psychology (5th ed.). Fort Worth, TX: Harcourt Brace Jovanovich.

Duckitt, J. H. (1983). Household crowding and psychological well-being in a South African colored community. The Journal of Social Psychology, 121, 275-282.

Gifford, R. (2007). Environmental Psychology, principles and practice (4th ed.). Colville, WA: Optimal Books. 
Khoury, H. T. T. (2005). Controle primário e controle secundário: Relação com indicadores de envelhecimento bem-sucedido. Tese de doutorado não-publicada, Universidade de Brasília.

Khoury, H. T. T., \& Günther, I. A. (2006). Percepção de controle, qualidade de vida e velhice bemsucedida. In D. V. da S. Falcão \& C. M. de S. B. Dias (Orgs.), Maturidade e velhice: Pesquisas e intervenções psicológicas (Vol. 2, pp.297-312). São Paulo: Casa do Psicólogo.

Lawton, M. P. (1983). Environmental and other determinants of well-being in older people. The Gerontologist, 23, 349-357.

Lerner, L. G. (2001). A arquitetura como disciplina coadjuvante no cuidado a pessoas com confusão mental e idosos dependentes. Cadernos IPUB/ Instituto de Psiquiatria da UFRJ, 10, 229-241.

Oliver, K. (2002). Psychology in practic: Environment. London: Hodder \& Stoughton.

Perracini, M. R. (2006). Planejamento e adaptação do ambiente para pessoas idosas. In E. V. de Freitas, L. Py, F. A. X. Cançado, J. Doll \& M. L. Gorzoni (Orgs.), Tratado de geriatria $e$ gerontologia (pp.1142-1151). Rio de Janeiro: Guanabara Koogan.

Pynoos, J., Nishita, C., \& Perelman, L. (2003). Advancements in the home modification field: A tribute to M. Powell Lawton. In R. J. Scheidt \& P. G. Windley (Eds.). Physical environments and aging: Critical contributions of M. Powell Lawton to theory and practice. New York: The Haworth Press.

Seligman, M. E. P., \& Csikszentmihalyi, M. (2000). Positive psychology. American Psychologist, 55, 5-14.

Sinha, S. P., \& Nayyar, P. (2000). Crowding effects of density and personal space requirements among older people: The impact of self-control and social support. The Journal of Social Psychology, 140, 721-728.
Artigo recebido em 10/12/2007.

Aceito para publicação em 28/04/2008.

Este trabalho é parte da Tese de Doutorado da primeira autora, orientada pela segunda autora e defendida na Universidade de Brasília, em 2005, sob o título "Controle primário e controle secundário: relação com indicadores de envelhecimento bemsucedido".

Endereço para correspondência:

Hilma Tereza Tôrres Khoury. Universidade Federal do Pará. Instituto de Filosofia e Ciências Humanas. Faculdade de Psicologia. Campus Universitário do Guamá. Rua Augusto Corrêa, 01. CEP: 66.075-110. Belém-PA, Brasil. E-mail: hilmatk@yahoo.com.br

Hilma Tereza Tôrres Khoury é Professor Associado do Instituto de Filosofia e Ciências Humanas da Universidade Federal do Pará.

Isolda de Araújo Günther é pesquisadora do Programa de Pós-graduação em Psicologia Escolar e do Desenvolvimento da Universidade de Brasília. 